\title{
High Sensitive C Reactive Protein (hsCRP) and Alkaline Phosphatase (ALP) in Type 2 Diabetes Patients
}

Nazrana Martuza ${ }^{1 *}$, Rezwana Haque ${ }^{2}$, Shamima Afrin ${ }^{3}$, Abu Jafar Md Tareq Morshed ${ }^{4}$, Afia Shahnaj ${ }^{5}$, Md. Tufazzal Hossain $^{6}$, Mohammad Shiblee Zaman ${ }^{7}$

\author{
${ }^{1}$ Assistant Professor, Department of Biochemistry, Ashiyan Medical College, Dhaka, Bangladesh \\ ${ }^{2}$ Assistant Professor, Department of Biochemistry, Uttara Adhunik Medical College, Dhaka, Bangladesh \\ ${ }^{3}$ Assistant Professor, Department Of Biochemistry and Molecular Biology, Sir Salimullah Medical College \& Mitford Hospital, \\ Dhaka, Bangladesh \\ ${ }^{4}$ Assistant Registrar, Cardiac Surgery, National Institute of Cardiovascular Diseases (NICVD), Dhaka, Bangladesh \\ ${ }^{5}$ Assistant Professor, Biochemistry, Dhaka Medical College, Dhaka, Bangladesh \\ ${ }^{6}$ Senior Biochemist, Popular Diagnostic Centre, Badda, Dhaka, Bangladesh \\ ${ }^{7}$ Assistant Professor, Biochemistry, Sunamganj Medical College, Sunamganj, Bangladesh
}

DOI: $10.36348 /$ sijb.2022.v05i01.004 | Received: 13.12.2021 | Accepted: 25.01 .2022 | Published: 30.01 .2022

*Corresponding author: Nazrana Martuza

Assistant Professor, Department of Biochemistry, Ashiyan Medical College, Dhaka, Bangladesh

\section{Abstract}

Background: Diabetes is a group of metabolic disorders, characterized by hyperglycemia which results from defects in insulin secretion, insulin action, or both. The prevalence and incidence of type 2 diabetes are rising rapidly worldwide. Diabetes has been linked to a shorter life expectancy mainly because of its complications, including heart disease, strokes, retinopathy, and chronic kidney disease and bone disease. Objective: To examine the relationship between high sensitive $\mathrm{C}$ reactive protein (hsCRP) and alkaline phosphatase (ALP) in type 2 diabetic patients. Methods: This study was a hospital based cross sectional study conducted at Dept. of Biochemistry, Ashiyan Medical College Hospital from January to June 2020. The study consists of 180 patients out of which 90 were normal healthy controls (Group I) and 90 patients having type 2 DM were included in case (Group II). FBS, PPBS, HbA1c, hsCRP and ALP of all subjects were measured. Results: Mean serum hsCRP and ALP level were statistically significant higher in case group compared to control group. Moreover, significant positive correlation was observed between hsCRP and ALP level as well as both with FBS, PPBS and HbA1c. Conclusions: Oxidative stress and inflammation appears to be a key component and also associated with poor glycaemic control and further pathogenesis of diabetes and its complications. All our finding suggest a link between oxidative stress, inflammation and glycaemic control in patient with type 2 diabetes mellitus.

Keywords: C - reactive protein, Alkaline Phosphatase, Diabetes, Mellitus, Post Prandial Blood Sugar.

Copyright (C) 2022 The Author(s): This is an open-access article distributed under the terms of the Creative Commons Attribution 4.0 International License (CC BY-NC 4.0) which permits unrestricted use, distribution, and reproduction in any medium for non-commercial use provided the original author and source are credited.

\section{INTRODUCTION}

The International diabetic federation (IDF) estimated in 2011 that 366 million adults aged between 20 - 79 years of the world's 7 billion population have diabetes mellitus. This gives a comparative prevalence of $8.5 \%$. Since more than $90 \%$ of the global cases of Diabetes mellitus are type 2 , it is evident that the prevalence was mainly due to the escalation of the causes of type 2 Diabetes mellitus. According to the current definition, two fasting glucose measurements above or equal to $126 \mathrm{mg} / \mathrm{dl}(7.0 \mathrm{mmol} / \mathrm{L})$ are considered diagnostic for Diabetes mellitus or Glycated Hemoglobin (HbA1C) above 6.5\% [1]. Diabetes
Mellitus is a group of metabolic disorders which is characterized by hyperglycemia due to the defects in insulin secretion, action of insulin, or both. The chronic hyperglycemia of diabetes mellitus is linked with longterm damage, disordered function and failure of different organs, especially the eyes, kidneys, nerves, heart, and blood vessels [2]. Diabetes mellitus (DM) is a clinical syndrome characterized by abnormal metabolism of carbohydrate, protein and fat resulting in hyperglycemia due to absolute or relative deficiency of insulin ending up in vascular complications leading to retinopathy, neuropathy and nephropathy. It can be divided into two main categories. Insulin Dependent 
Diabetes Mellitus (IDDM) now labeled as type-1 Diabetes Mellitus and Non-Insulin Dependent Diabetes Mellitus (NIDDM) known as type-2 Diabetes Mellitus (type 2 DM). Diabetes mellitus (DM) comprises a group of common metabolic disorders that share common phenotype of hyperglycemia [2]. Hyperglycemia not only defines the disease but is the cause of its most characteristic symptoms and long-term complications. Understanding the pathogenesis and preventing long-term complications have been major goals of research in diabetes mellitus. Research in the past few years has linked inflammation to $\beta$-cell dysfunction resulting from chronic exposure to hyperglycemia. A growing body of data reinforces the concept that inflammation plays an important role in the pathogenesis of type $2 \mathrm{DM}$ and links DM with concomitant conditions with inflammatory components [3]. Alkaline phosphatase (ALP) is a hydrolase enzyme, which is widely expressed in human tissues, but is highly concentrated in the liver, bone, and kidney [4, 5]. Physiological increases are found during bone growth, while pathological increases are largely associated with hepatobiliary and bone diseases. Type-2 Diabetes Mellitus constitutes $85-90 \%$ of diabetic patients. Uncontrolled diabetes (chronic hyperglycemia) is associated with several long-term complications, related with micro-vascular diseases including Retinopathy, nephropathy, neuropathy and macro vascular diseases such as cardiovascular and cerebro vascular, increased susceptibility to infection and poor wound healing. It has been reported that many diabetics may also exhibit elevated serum alkaline phosphatase level. Alkaline phosphatase is an inflammatory mediator like Creactive protein (CRP) (a novel risk marker for cardiovascular disease) [6]. Both ALP and CRP have consistently been shown to be directly and significantly associated with each other, with suggestions that they share common biological pathways [7]. Over the past decade, serum ALP has sparked interest as an emerging marker for cardiovascular risk in the general population, but uncertainty exists because important questions pertaining to its association with CVD remain unresolved. High sensitivity C-reactive protein (hsCRP) is a $\mathrm{C}$-reactive protein measured by a highly sensitive assay. CRP represents the classical acute-phase protein produced in the liver in response to inflammatory stimuli, and plasma levels of hsCRP provide a sensitive marker of increased inflammatory activity in the arterial wall $[8,9]$. Chronic, systemic subclinical inflammation has also been identified as a driving force for insulin resistance, metabolic syndrome and type $2 \mathrm{DM}$. High sensitivity C-reactive protein (hsCRP) is a C-reactive protein measured by a highly sensitive assay. CRP represents the classical acute-phase protein produced in the liver in response to inflammatory stimuli, and plasma levels of hsCRP provide a sensitive marker of increased inflammatory activity in the arterial wall [8, 9]. Because the development of complications is linked to the accumulation of glycation adducts in tissue proteins. The core of the issue is glycemic control.
Optimal monitoring of glycemic control involves plasma glucose measurements (fasting and postprandial blood sugar) and measurement of glycated hemoglobin (HbA1c). Since the inflammation appears to be a key component of many reactions associated with poor glycemic control and further pathogenesis of diabetes and its complications; we found it interesting to study serum ALP activity (marker of CVD) and hsCRP level (an inflammatory marker) in diabetic subjects.

\section{MATERIALS AND METHODS}

This study was a hospital based cross sectional study conducted at Dept. of Biochemistry, Ashiyan Medical College Hospital from January to June 2020. The study consisted of 180 subjects among whom 90 persons were healthy controls and 90 patients were Type $2 \mathrm{DM}$ were taken as cases.

\section{Inclusion Criteria}

The subjects selected for study were grouped as follows: Group I - Control group ( $=90)$ : This group consisted of age and gender matched healthy subjects. They were taken from general population who came for routine checkup. Group II - Case group - Type 2 DM patients $(n=90)$ as ADA diagnostic criteria.

\section{Exclusion Criteria}

The patients with type 1 diabetes mellitus, high $(>120 \mathrm{~g} / \mathrm{d})$ alcohol consumption, with known liver or gastrointestinal diseases, with liver enzyme concentrations higher than three times the upper limit, on corticosteroids, methotrexate, amiodarone, tamoxifen or other hepatotoxic drugs, any chronic infection like tuberculosis, sarcoidosis and hemolytic anemia were excluded from this study.

\section{Sample Collection and Analysis}

A $5 \mathrm{ml}$ of venous blood was drawn from each volunteer using a disposable vacationer system in fasting condition (Plain, EDTA and Fluoride). Serum or plasma separated within half an hour and stored at 2$8^{\circ} \mathrm{C}$ temperature till analysis was done. Analysis of sample Fasting and post prandial (2 hour) blood sugar (FBS \& PPBS) estimated by glucose oxidaseperoxidase (GOD-POD) enzymatic end point method (Kit: Quantitative determination blood sugar by glucose oxidase peroxidase method cobas integra). Glycated hemoglobin $(\mathrm{HbA1c})$ Concentration was measured by immuno turbidimetric method. Serum ALP activity was determined by carboxy substrate kinetic method. (Kit: Quantitative determination of ALP by carboxyl substrate method cobas integra). Serum hsCRP level is measured by immuno turbidimetric method (Kit: Quantitative determination of hsCRP in human blood by latex turbidity assay cobas integra).

\section{Data Collection \& Statistical Analysis}

Demographic data, laboratory result and clinical details of all potential candidates were recorded 
in an especially designed questionnaire. Un-paired t-test was applied to analysed the study data.

\section{RESULTS}

In our study characteristics of the study population shows the demographic and clinical characteristics of the case and control groups. Baseline characteristics of case and control group as well other biochemical parameter are given in (Table 1). Baseline characteristics like age and gender in case and control group were not significant difference. Whereas, there were significant differences in BMI, waist circumference (WC), hip circumference (HIP), waistto-hip ratio (WHR), Systolic blood pressure (SBP) and diastolic blood pressure (DBP) between case and Control groups ( $\mathrm{p}$ values $<0.001)$.

Table-1: Comparison of baseline characteristics between case and control groups $(\mathbf{N}=180)$

\begin{tabular}{|l|l|l|l|}
\hline & $\begin{array}{l}\text { Case }(\mathbf{n = 9 0}) \\
\text { Mean } \pm \text { SD }\end{array}$ & $\begin{array}{l}\text { Control }(\mathbf{n = 9 0}) \\
\text { Mean } \pm \text { SD }\end{array}$ & p - value \\
\hline Age & $53.33 \pm 8.23$ & $49.21 \pm 7.01$ & 0.78 \\
\hline Sex $($ Male/female) & $61 / 29$ & $54 / 36$ & \\
\hline BMI $(\mathrm{kg} / \mathrm{cm} 2)$ & 26.9 & 23.1 & $<0.001$ \\
\hline WC $(\mathrm{cm})$ & $89.21 \pm 8.21$ & $82.92 \pm 6.93$ & $<0.001$ \\
\hline HIP $(\mathrm{cm})$ & $94.94 \pm 7.23$ & $92.14 \pm 6.42$ & $<0.001$ \\
\hline WHR & 0.94 & 0.85 & $<0.001$ \\
\hline SBP $(\mathrm{mmHg})$ & $137 \pm 14$ & $123 \pm 13$ & $<0.001$ \\
\hline DBP $(\mathrm{mmHg})$ & $89 \pm 11$ & $81 \pm 9$ & $<0.001$ \\
\hline
\end{tabular}

Table-2: Comparison between case and control group ( $N=180)$

\begin{tabular}{|l|l|l|l|}
\hline & Case & Control & p - value \\
\hline FBS $(\mathrm{mg} / \mathrm{dl})$ & $168.03 \pm 22.45$ & $96.21 \pm 14.45$ & $<0.001$ \\
\hline PPBS $(\mathrm{mg} / \mathrm{dl})$ & $194.78 \pm 29.53$ & $121.67 \pm 18.34$ & $<0.001$ \\
\hline HbA1c $(\%)$ & $7.21 \pm 0.83$ & $5.89 \pm 0.41$ & $<0.001$ \\
\hline hsCRP $(\mathrm{mg} / \mathrm{dl})$ & $3.17 \pm 0.61$ & $1.21 \pm 0.23$ & $<0.001$ \\
\hline
\end{tabular}

Table-3: Values of serum hsCRP and ALP concentration between case and control group ( $N=180)$

\begin{tabular}{|l|l|l|}
\hline & hsCRP $(\mathbf{m g} / \mathbf{d l})$ & ALP $($ IU/L) \\
\hline Case & $3.17 \pm 0.61$ & $151 \pm 19.54$ \\
\hline Control & $1.21 \pm 0.23$ & $139 \pm 16.23$ \\
\hline t- value & 31.23 & 21.43 \\
\hline P value & $<0.0001$ & $<0.0001$ \\
\hline
\end{tabular}

Table-4: Pearson's correlation analysis between serum hsCRP, ALP and glycaemic control (N=180)

\begin{tabular}{|l|l|l|}
\hline & Correlation coefficient r value & Two tailed p value \\
\hline Serum hsCRP with ALP & 0.32 & $<0.0001$ \\
\hline Serum hsCRP with FBS & 0.37 & $<0.0001$ \\
\hline Serum hsCRP with PPBS & 0.36 & $<0.0001$ \\
\hline Serum hsCRP with HbA1c & 0.43 & $<0.0001$ \\
\hline Serum ALP with FBS & 0.33 & $<0.0001$ \\
\hline Serum ALP with PLBS & 0.41 & $<0.0001$ \\
\hline Serum ALP with HbA1c & 0.72 & $<0.0001$ \\
\hline
\end{tabular}

Our study shows that mean serum FBS level in case group was significantly higher compared to control ( $\mathrm{p}$ values < 0.001). Also, PPBS level in case was significantly higher compared to control group ( $\mathrm{p}$ values < 0.001). Furthermore, mean $\mathrm{HbA} 1 \mathrm{c} \%$ in case were significantly higher compared to control ( $\mathrm{p}$ values $<$ 0.001). But serum alkaline phosphatase (ALP) concentration is not increased between case and control groups ( $p$ value is 0.71 which is considered not significant). In addition, mean hsCRP levels in case were significantly higher compared to control group ( $\mathrm{p}$ values < 0.001) (Table 2). There were significant differences in serum hs-CRP and ALP levels between case and control groups (both $\mathrm{p}<0.0001$ ). Compared to controls group, there were significantly higher hs-CRP levels and serum ALP in the case group (both p < 0.001) ((Table 3). Correlations of hs-CRP levels with clinical and laboratory parameters between case-control groups: Univariate correlation analysis demonstrated that statistically significant correlations of hs-CRP with other clinical and quantitative laboratory parameters were found (all $\mathrm{p}<0.0001$ ). In addition there was a statistically significant positive correlation of serum ALP levels with hs-CRP and glycaemic triad (FBS, PPBS and HbA1c) in the case group (all p < 0.0001) (Table 4). 


\section{DISCUSSION}

This study was correlate serum hs-CRP, serum ALP and glycaemic triad (FBS, PPBS and HbA1c) in case and control group. Our study shows that statistically significant increased concentration of hsCRP and ALP in patients with case group (type 2 DM) compared with control group (healthy persons). Also, we found a significant positive linear relationship between hs-CRP and ALP concentration as well as both with FBS, PPBS and HbA1c. These findings suggest a link between inflammations (raised hsCRP concentration), oxidative stress (indicated by increased serum ALP concentration) and glycaemic control in patients with type $2 \mathrm{DM}$ and related complications. Moreover, concentration of hs-CRP and ALP were significantly elevated in patients with type 2 DM compared to healthy subjects. Glycaemic triad such as FBS, PPBS and HbA1c showed a significant rise in type 2 diabetes then control group. It can be seen that there is significant increase of HbAlc in diabetics than non-diabetics. Several possible mechanisms which explain increased FBS, PPBS and HbA1c concentration in patients with type $2 \mathrm{DM}$ and its correlation with glycaemic control. Sarinnapakorn V, et al. found that hsCRP levels correlated with HbA1c levels [12]. Mean HbA1c levels were significantly higher in patients who had more hsCRP levels. Other factors such as age, BMI, blood pressure, screenings correlated with hsCRP level. Because of a positive correlation between serum hsCRP and FBS, PPBS, HbAlc, and inflammation, insulin resistance and hyperglycaemia jointly contribute to the cardiovascular risk in type-2 DM. This suggests a role of oxidative stress and chronic low-grade inflammation in pathogenesis of type 2 diabetes patients [13]. There are various studies which support our results. R Sharma et al., shows rise in levels of hsCRP and ALP in diabetic subjects and their significant association which might be a result of inflammation in diabetes mellitus [14]. Ahmed Khan D et al., studied diabetic patients had significantly elevated median of HbA1c, hsCRP and GGT as compared to controls [15], HbA1c showed a positive correlation with hsCRP, ALP and inflammatory markers used in addition to $\mathrm{HbA} 1 \mathrm{c}$ for assessment of increased risk in diabetic patients because of accelerated atherosclerosis due to free radical injury[16]. In the present study, our results showed a significantly higher hs-CRP level in the type 2 diabetes patients than in normal controls. Serum hsCRP is positively associated with the metabolic syndrome and has been acknowledged to be an independent risk factor for development of diabetic neuropathy, diabetic foot ulcers and CV complications [17]. Recently, Aryan et al., performed a larger sample population-based study to evaluate the predicted value of hs-CRP for complications of T2DM [18]. Hs-CRP has been applied in clinical settings to monitor chronic and acute inflammatory conditions [19]. In addition, it has also been reported that hs-CRP has an association with insulin resistance, of which CRP may contribute to vascular inflammation and cause injury of vascular cells and further contribute to the development of insulin resistance [20]. Our results also suggest that liver enzymes are closely associated with the risk of metabolic syndrome and type 2 diabetes and that among this serum ALP is the most powerful risk indicator for developing the metabolic syndrome and type 2 diabetes. Similar study conducted by Vazarova et al stated that serum ALP levels were mildly increased in Type 2 diabetes patients [21]. Possible pathophysiological mechanism is that elevated liver enzymes may reflect due to obesity, fatty liver, hepatosteatosis, inflammation, which impairs insulin signalling both in the liver and systemically [22]. Elevation of serum ALP could be the expression of an excess deposition of fat in the liver, termed non-alcoholic fatty liver disease. Fatty liver is thought to cause hepatic insulin resistance and to contribute to the development of systemic insulin resistance and hyper insulinemia [23]. Elevated Serum ALP level may be a simple and reliable marker of visceral and hepatic fat and, by inference, of hepatic insulin resistance. In addition, implication of inflammatory in the pathogenesis of diabetes is suggested, not only by oxygen free-radical generation, but also due to non-enzymatic protein glycosylation, autoxidation of glucose, impaired glutathione metabolism [24]. Increases in ALP activity can be a response to oxidative stress, facilitating increased transport of GSH precursors into cells. In addition, ALP is leaked into the serum possibly as a result of normal cell turnover and cellular stresses. These findings suggest that a raised serum ALP level is an independent risk factor for type 2 diabetes [25]. Despite these potential limitations, our findings, which were obtained from a cross sectional study shows that serum ALP activity and hsCRP level is significantly increased in patients with type 2 diabetes mellitus compared to healthy control. Both are further increased in diabetic patients with complications. Also, there is a significant positive correlation between serum ALP activity and hsCRP. Both are also independently positively correlated with HbA1c, FBS and PPBS (short and long term glycaemic control). So far, the underlying pathophysiological mechanisms due to insulin resistance, oxidative stress and chronic low grade systemic inflammation may be involved. All these finding suggesting a link between oxidative stress, inflammation and glycaemic control in patient with type 2 diabetes mellitus. Further studies are needed to investigate the biological mechanisms underlying this association.

\section{CONCLUSION}

In concluded, we investigated the serum FBS, PPBS, HbA1c, hs-CRP and ALP concentrations in Type 2DM and their relations with clinical and laboratory features. Although FBS, PPBS, HbA1c, hsCRP and ALP have been studied in many metabolic diseases, there are very few studies regarding the expression of FBS, PPBS, HbA1c, hs-CRP and ALP and their association with Type 2 DM. The current 
study demonstrated that, in comparison to healthy controls, there were significantly increased FBS, PPBS, HbA1c, hs-CRP and ALP levels in Type 2DM. Furthermore, hs-CRP level was correlated with BMI, ALP in the Type 2DM group, and was associated with FBS, PPBS and HbA1c. Serum ALP level and hsCRP concentration was independently and positively correlated with FBS, PPBS and HbA1c (markers of glycaemic control). All these finding suggesting a link between oxidative stress, inflammation and glycaemic control in patient with type 2 diabetes mellitus. There is a significant positive correlation between serum ALP activity and hsCRP.

\section{REFERENCES}

1. Hansen, D., Dendale, P.L., Beelen, M., Jonkers, A., Mullens, A., \& Coruy, L. (2010). Plasma adipokine and inflammatory marker concentrations are altered in obese, as opposed to non-obese, type 2 Diabetes patients. European Journal Applied Physiology, 109, 397-404. http://dx.doi.org/10.1007/s00421-010-1362-5

2. Alvin, C. (2005). Powers: diabetes mellitus. In Harrison's Principle of Internal Medicine.16th ed. Edited by Kasper, L. New York: McGraw-Hill.

3. Alvin, C. (2001). Powers. Diabetes mellitus. Harrison's principle of internal medicine, 16, 3779829.

4. Ford, E. S. (2003). The metabolic syndrome and Creactive protein, fibrinogen, and leukocyte count: findings from the Third National Health and Nutrition Examination Survey. Atherosclerosis, 168(2), 351-358.

5. Schoppet, M., \& Shanahan, C. M. (2008). Role for alkaline phosphatase as an inducer of vascular calcification in renal failure?. Kidney international, 73(9), 989-991.

6. Moss, D. W. (1994). Release of membrane-bound enzymes from cells and the generation of isoforms. Clinica chimica acta, 226(2), 131-142.

7. Löe, H. (1993). Periodontal disease: the sixth complication of diabetes mellitus. Diabetes care, 16(1), 329-334.

8. Enlow, D. H. (1999). Physiologic tooth movement and alveolar remolding. In Enlow DH, ed. Facial Growth. Philadelphia; Saunders, 130-48.

9. Biasi, D., Carletto, A., Dell'Agnola, C., Caramaschi, P., Montesanti, F., Zavateri, G., \& Bambara, L. M. (1996). Neutrophil migration, oxidative metabolism, and adhesion in elderly and young subjects. Inflammation, 20(6), 673-681.

10. Ainamo, J., Lahtinen, A., \& Uitto, V. J. (1990). Rapid periodontal destruction in adult humans with poorly controlled diabetes a report of 2 cases. Journal of Clinical Periodontology, 17(1), 22-28.

11. Sarinnapakorn, V., \& Wanicagool, W. (2013). Association between hs-CRP and Hbalc in overweight type 2 diabetic female patients. Journal of the Medical Association of Thailand= Chotmaihet thangphaet, 96, S54-8.
12. Svensson, A. M., Miftaraj, M., Franzén, S., \& Eliasson, B. (2017). Clinical effects, cardiovascular and renal outcomes associated with rapid-acting insulin analogs among individuals with type 2 diabetes: a nation-wide observational cohort study. Clinical diabetes and endocrinology, 3(1), 5.

13. Surendar, J., Anuradha, S., Ashley, B., Balasubramanyam, M., Aravindhan, V., Rema, M., \& Mohan, V. (2009). Cystatin C and cystatin glomerular filtration rate as markers of early renal disease in Asian Indian subjects with glucose intolerance (CURES-32). Metabolic syndrome and related disorders, 7(5), 419-425.

14. Khan, D. A., \& Qayyum, S. (2009). Evaluation of cardiac risk by oxidative stress and inflammatory markers in diabetic patients. Pak J Med Sci, 25(5), 776-81.

15. Raynor, L. A., Pankow, J. S., Duncan, B. B., Schmidt, M. I., Hoogeveen, R. C., Pereira, M. A., ... \& Ballantyne, C. M. (2013). Novel risk factors and the prediction of type 2 diabetes in the Atherosclerosis Risk in Communities (ARIC) study. Diabetes care, 36(1), 70-76.

16. Abbasi, A., Bakker, S. J., Corpeleijn, E., Gansevoort, R. T., Gans, R. O., Peelen, L. M., ... \& Beulens, J. W. (2012). Liver function tests and risk prediction of incident type 2 diabetes: evaluation in two independent cohorts. PloS one, 7(12), e51496.

17. Brunner, E. J., Kivimäki, M., Witte, D. R., Lawlor, D. A., Smith, G. D., Cooper, J. A., \& Shah, T. (2008). Inflammation, insulin resistance, and diabetes-Mendelian randomization using CRP haplotypes points upstream. PLoS medicine, 5(8), e155.

18. Bahceci, M., Tuzcu, A., Ogun, C., Canoruc, N., Iltimur, K., \& Aslan, C. (2005). Is serum Creactive protein concentration correlated with $\mathrm{HbA}$ $1 \mathrm{c}$ and insulin resistance in Type 2 diabetic men with or without coronary heart disease?. Journal of endocrinological investigation, 28(4), 145-150.

19. Yuan, G., Zhou, L., Tang, J., Yang, Y., Gu, W., Li, F., \& Chen, M. (2006). Serum CRP levels are equally elevated in newly diagnosed type 2 diabetes and impaired glucose tolerance and related to adiponectin levels and insulin sensitivity. Diabetes research and clinical practice, 72(3), 244-250.

20. Vozarova, B., Stefan, N., Lindsay, R. S., Saremi, A., Pratley, R. E., Bogardus, C., \& Tataranni, P. A. (2002). High alanine aminotransferase is associated with decreased hepatic insulin sensitivity and predicts the development of type 2 diabetes. Diabetes, 51(6), 1889-1895.

21. Sato, K. K., Hayashi, T., Nakamura, Y., Harita, N., Yoneda, T., Endo, G., \& Kambe, H. (2008). Liver enzymes compared with alcohol consumption in predicting the risk of type 2 diabetes: the Kansai Healthcare Study. Diabetes care, 31(6), 12301236. 
22. Rani, D. P., \& Anandan, S. N. (2012). A clinical study of serum alkaline phosphatase and calcium level in type 2 diabetes mellitus with periodontitis among the south Indian population. SRM Journal of Research in Dental Sciences, 3(3), 175.

23. Tang, F. T., Chen, S. R., Wu, X. Q., Wang, T. Q., Chen, J. W., Li, J., \& Liu, P. Q. (2006). Hypercholesterolemia accelerates vascular calcification induced by excessive vitamin D via oxidative stress. Calcified tissue international, 79(5), 326-339.

24. Varma, V., Varma, M., Varma, A., Kumar, R., Bharosay, A., \& Vyas, S. (2016). Serum total sialic acid and highly sensitive C-reactive protein: Prognostic markers for the diabetic nephropathy. Journal of laboratory physicians, 8(01), 025-029.

25. Kerner, A., Avizohar, O., Sella, R., Bartha, P., Zinder, O., Markiewicz, W., \& Aronson, D. (2005). Association between elevated liver enzymes and Creactive protein: possible hepatic contribution to systemic inflammation in the metabolic syndrome. Arteriosclerosis, thrombosis, and vascular biology, 25(1), 193-197. 